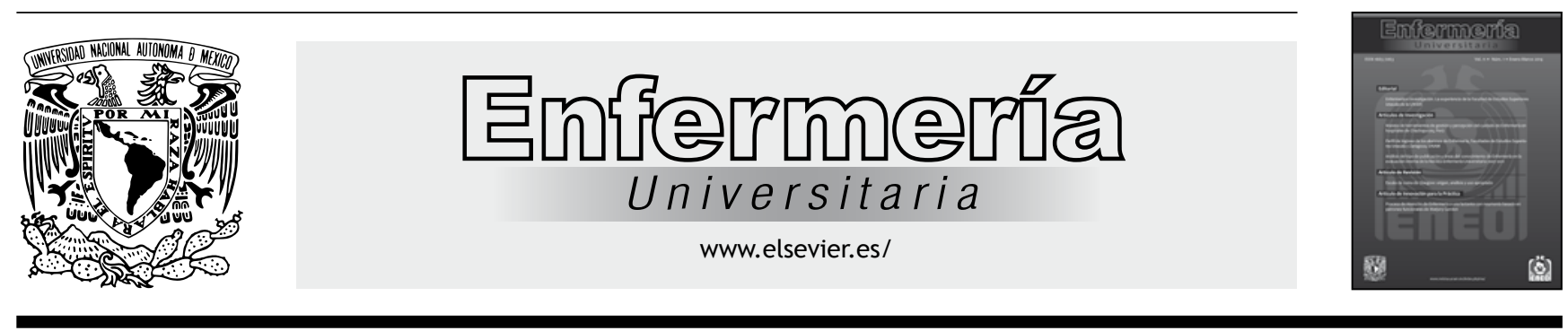

EDITORIAL

\title{
Enfermería e Investigación. La experiencia de la Facultad de Estudios Superiores Iztacala de la UNAM
}

\section{Nursing and Research. The experience of the Faculty of Iztacala UNAM}

La investigación en Enfermería que actualmente se realiza en la Facultad de Estudios Superiores Iztacala puede ser considerada como en desarrollo, esto tiene que ver, por un lado, con la evolución del Programa Institucional de Investigación y por otro, por el propio devenir histórico de la $\mathrm{Ca}$ rrera de Enfermería en esta Facultad.

En el año 1975 a raíz del Programa de Expansión de la UNAM se crea la entonces Escuela Nacional de Estudios Profesionales Iztacala (ENEPI) donde se albergan las Carreras de Biología, Medicina, Cirujano Dentista, Psicología y Enfermería (esta última en nivel técnico). Inicialmente, la investigación en la disciplina se vinculaba a la práctica de Enfermería en el trabajo que se realizaba en el ámbito comunitario y hospitalario, sin ser todavía formalmente instituida a través de un Programa de Investigación.

En el año 1990, acorde a las funciones sustantivas de la UNAM, se observa la necesidad de organizar y promover un espacio académico donde los profesores e investigadores de las diferentes áreas pudieran interactuar en un trabajo interdisciplinario. Derivado de lo anterior se promueve la creación de Proyectos Troncales de Investigación por Carrera a fin de profundizar en los aspectos teóricos, metodológicos y temáticos relacionados con los procesos de investigación lo que permitiría, con los resultados obtenidos en dichas investigaciones, retroalimentar la vida escolar y comunitaria desde las Carreras ${ }^{1}$.

En esta primera etapa del Proyecto Troncal de Investigación se determinó que las áreas de interés serían aquellas en las cuales los profesionales de Enfermería desarrollaban su práctica: la clínica y la de salud pública.

Para ese momento histórico, la Carrera de Enfermería era la única de nivel técnico en el campus y no contaba con profesores de Carrera sólo de asignatura, en su mayoría contratados por horas, situación que más adelante llevó a generar plazas de profesor de Carrera (laboralmente estos profesionales son los responsables de generar conocimiento a través de la investigación). Para quienes aspiraban a ocupar estas plazas fue necesario formarse en los niveles de licenciatura y luego en el posgrado.

Los resultados obtenidos en un Taller de Evaluación Curricular de los años 1985-1994, evidenciaron la necesidad de fortalecer el proyecto académico de la Carrera e impulsar el crecimiento de su personal docente, elementos que fundamentaron la apertura de una tercera área de investigación dentro del Proyecto Troncal: la educativa.

Un hecho sin duda importante es que gracias a los trabajos realizados el 14 de mayo de 2001, la ENEP Iztacala se transforma en Facultad denominándose FESI, Facultad de Estudios Profesionales Iztacala².

Para el año 2002 el Proyecto Troncal, se estructuró como Programa de Investigación y en ese momento se integran las líneas de investigación mejor definidas y consideradas específicas para la Carrera, buscando también una correspondencia con el Plan de Desarrollo Académico Institucional (PDAI) de la FESI, que incluía los Programas de Fortalecimiento para la Investigación y Superación y la incorporación del profesor de Carrera a la investigación.

En ese mismo año, la Carrera de Enfermería retoma la experiencia de los años anteriores y propone establecer el Programa de Investigación con base en el desarrollo curricular hasta ese momento así como las necesidades de la Facultad, de la disciplina y de la sociedad.

Habiendo seguido el proceso instituido, el plan de estudios de la Licenciatura en Enfermería, con un nuevo diseño Curricular, inició su implementación en el ciclo escolar 2003.

Para el año 2006 surge el Programa Rector de Investigación, donde se marcan las subdisciplinas de educación, 
salud y epidemiología con líneas definidas, las cuales han contribuido al aporte de conocimiento.

Un hecho trascendental para la investigación es la participación de la Carrera de Enfermería dentro del Programa de Maestría en Enfermería (en conjunto con la FES Zaragoza y la ENEO), el cual inicia su implementación en el año 2004, lo que posibilitó que profesores de Carrera sean, hasta la actualidad, asesores de Tesis de alumnos(as) de este Programa.

En este momento la plantilla de la Carrera de Enfermería cuenta con 8 profesores de carrera asociados y 3 titulares los cuales realizan investigación de acuerdo a las líneas del Programa Rector a través de definir y vincular la participación en el proyecto de investigación de la Carrera, con el apoyo y fortalecimiento de profesores y alumnos, así como de los diversos Programas Institucionales de financiamiento a proyectos de investigación que la Universidad ofrece.

Para alcanzar lo anterior el grupo está reestructurando las líneas de investigación y el Programa Rector, dada la importancia de incrementar el saber de la propia disciplina, aspecto que puede coadyuvar en la autonomía del saber de Enfermería así como abonar al reconocimiento social del cuidado de Enfermería y la diferenciación de la atención médica, el enfoque está enmarcado en el objeto de estudio de la Enfermería: el cuidado.

Las aportaciones de las investigaciones que actualmente realizan los profesores de la Carrera de Enfermería de la FESI, buscan apoyar los conocimientos sobre el cuidado de la persona a través de la descripción, el análisis, la explicación y la comprensión de los fenómenos que afectan la salud individual y colectiva como un medio a través del cual se logra la meta específica del profesional en el mejoramiento de la práctica ${ }^{3}$. Lo anterior, también posibilita ir perfilando a la Enfermería para alcanzar el estatus de disciplina científica, profesión transdisciplinar del campo de las ciencias sociales y de la salud, cuyo objeto de estudio e intervención es el cuidado humano ${ }^{4}$.

Con base en este recuento histórico podemos asegurar que aunque sea un grupo pequeño y joven en la investigación, las(os) profesores que realizan investigación y que forman parte del grupo de trabajo de la Carrera de Enfermería de la FESI, han avanzado a través de realizar aportaciones al conocimiento de Enfermería, tomando como base las líneas de investigación vigentes, y continuarán realizándolo con la nueva reestructuración de las mismas. Un hecho significativo es la difusión del conocimiento a través de diversas publicaciones en revistas nacionales e internacionales, contribuyendo al crecimiento y desarrollo científico de la profesión de Enfermería.

\author{
M. Acevedo-Peña \\ Jefe de la Carrera de Enfermería FESI \\ México D.F., México
}

\section{Referencias}

1. Pineda Olvera J. Rodríguez Zamora C. Programa rector de investigación. FESI. UNAM: México; 2006. p. 12.

2. AHFIZ. Esbozo histórico de la FES Iztacala UNAM. México 2012. http://docs.com/KFON

3. Durán de Villalobos M. Enfermería Desarrollo teórico e investigativo. Colombia: Universidad Nacional de Colombia; 2001. p. 25.

4. OPS. Orientaciones para la Educación Inicial de Enfermería en las Américas: hacia el 2020 - Documento en proceso de elaboración. OPS/OMS. HSS/HR/Enf. Washington, 24 de septiembre de 2007. 